\title{
Explicit Solutions of the Invariance Equation for Means
}

\author{
Janusz Matkowski, Monika Nowicka and Alfred Witkowski
}

\begin{abstract}
Extending the notion of projective means we first generalize an invariance identity related to the Carlson log given in Kahlig and Matkowski (Math Inequal Appl 18(3):1143-1150, 2015), and then, more generally, given a bivariate symmetric, homogeneous and monotone mean $M$, we give explicit formula for a rich family of pairs of $M$-complementary means. We prove that this method cannot be extended for higher dimension. Some examples are given and two open questions are proposed.
\end{abstract}

Mathematics Subject Classification. 26D15.

Keywords. Invariant means, homogeneous means.

\section{Introduction}

A function $\Phi: X \rightarrow Y$ is called invariant with respect to a selfmap $T: X \rightarrow X$ (briefly, $T$-invariant) if $\Phi \circ T=\Phi$. Invariant functions appear in iteration theory and fixed point theory. For instance, if $X$ is a metric space, $T$ is continuous and the sequence $\left(T^{n}\right)_{n \in \mathbb{N}}$ of iterates of $T$ is pointwise convergent, then the function $\Phi(x)=\lim _{n \rightarrow \infty} T^{n}(x)$ is $T$-invariant. A model illustration offer the mean-type mappings, i.e. the mappings of the form $(K, L)$, where the coordinate functions $K, L$ are bivariate means. Some conditions guarantying convergence of iterates $(K, L)^{n}$ to a unique $(K, L)$-invariant mean-type mapping $(M, M)$, and $M \circ(K, L)=M([7]$, also [4,6,11]), generalize in particular, the well-known theorem of Gauss [2] on the arithmetic-geometric iterations. If the invariance equality $M \circ(K, L)=M$ is satisfies one says that the means $K$ and $L$ are mutually $M$-complementary with respect to $M$ (briefly, $M$-complementary) [5].

It happens quite exceptionally, when a given mean-type mapping $(K, L)$ one can find the explicit form of the $(K, L)$-invariant mean. The identity $\mathrm{G} \circ$ $(A, H)=G$, where $A, G, H$ denote, respectively, arithmetic, geometric and 
harmonic mean, (equivalent to the classical Pythagorean harmony proportion $\left.\frac{\mathrm{A}}{\mathrm{G}}=\frac{\mathrm{G}}{\mathrm{H}}\right)$, meaning that $\mathrm{G}$ is $(\mathrm{A}, \mathrm{H})$-invariant, and allowing to conclude that

$$
\lim _{n \rightarrow \infty}(A, H)^{n}(x, y)=(G(x, y), G(x, y)) \quad x, y>0,
$$

is an example.

In these circumstances it is natural to ask if, a given mean $M$, one can find effectively some nontrivial pairs of $M$-complementary means. This problem appeared in connection with [3] where the authors proved that for $t \in[-1,1]$, $t \neq 0$, the unsymmetric means

$$
K_{t}=t x^{t} \frac{x-y}{x^{t}-y^{t}} \quad \text { and } \quad L_{t}=t y^{t} \frac{x-y}{x^{t}-y^{t}}
$$

are mutually complementary with respect to the logarithmic mean L, i.e. they satisfy the invariance equation

$$
\mathrm{L} \circ\left(K_{t}, L_{t}\right)=\mathrm{L} .
$$

In Sect. 3, we extend the notion of projective means by creating $2^{\mathfrak{c}}$ pairs of means $\left(\mathrm{P}_{A}, \mathrm{P}_{A^{\prime}}\right)$ satisfying $\left\{\mathrm{P}_{A}(x, y), \mathrm{P}_{A^{\prime}}(x, y)\right\}=\{x, y\}$, so that the functions

$$
K_{t, A}(x, y)=t \mathrm{P}_{A}^{t}(x, y) \frac{x-y}{x^{t}-y^{t}}
$$

and

$$
L_{t, A}(x, y)=t \mathrm{P}_{A^{\prime}}^{t}(x, y) \frac{x-y}{x^{t}-y^{t}}
$$

are L-complementary means. We also give necessary and sufficient conditions for these means to be symmetric and/or homogeneous, thus we provide answer to a question posed in [3].

In Sect. 4 , following the ideas of $[3,12]$, we prove that if $M: \mathbb{R}_{+}^{2} \rightarrow \mathbb{R}_{+}$ is a monotone, homogeneous and symmetric mean, then for every $t \in(-1,1)$ the function $M_{t}$ given by

$$
M_{t}(x, y)=\left(\frac{M(x, y)}{M\left(x^{t}, y^{t}\right)}\right)^{\frac{1}{1-t}}
$$

is a mean and the functions

$$
K_{t}(x, y)=x^{t} M_{t}^{1-t}(x, y) \quad \text { and } \quad L_{t}(x, y)=y^{t} M_{t}^{1-t}(x, y)
$$

are homogeneous $M$-complementary means. Actually, a stronger result holds true, namely the functions $K_{t, A}=\mathrm{P}_{A}^{t} M_{t}^{1-t}$ and $L_{t, A}=\mathrm{P}_{A^{\prime}}^{t} M_{t}^{1-t}$ are homogeneous $M$-complementary means. The construction the means $K_{t}, L_{t}$ depends on $M$, and they inherit the assumed symmetry and homogeneity of $M$, but in general, the monotonicity is not hereditary.

In Sect. 5 we ask whether the projective means considered in previous sections can be replaced by some other means. The examples constructed show that in even in case of very classical means $M, C, D$ the function 
$N_{t}=\left(\frac{M}{M \circ\left(C^{t}, D^{t}\right)}\right)^{\frac{1}{1-t}}$ may not be a mean. Nevertheless, we prove rather surprising fact, that if $M$ is a symmetric, homogeneous, monotone mean, $C, D$ are arbitrary means and $t \in(0,1)$, then the functions $K_{t}=C^{t} N_{t}^{1-t}, L_{t}=D^{t} N_{t}^{1-t}$ are $M$-complementary means.

Thus, the applied method gives explicit formulas for complementary means in case of monotone, symmetric and homogeneous means $M$; moreover the means $K_{t}$ and $L_{t}$ inherit the symmetry and/or homogeneity from $C$ and $D$. Homogeneity of $M$ is crucial here. We conclude that section with two open questions, and one of them is whether the monotonicity condition can be relaxed?

Noting that all the presented results have their translative counterparts, we formulate in Sect. 7 the translative counterpart of Theorem 3. An application of this result for the arithmetic mean $A$ gives all possible pairs of A-complementary weighted arithmetic means.

Finally in Sect. 8 we discuss the invariance equation for subtranslative and subhomogeneous means.

\section{Preliminaries}

The set of positive real numbers is denoted by $\mathbb{R}_{+}$. A mean is a function $M: \mathbb{R}_{+}^{2} \rightarrow \mathbb{R}_{+}$satisfying

$$
\min \{x, y\} \leq M(x, y) \leq \max \{x, y\} .
$$

Depending on additional properties a mean is called

strict

symmetric if $M(x, y)=M(y, x)$ for all $x, y$,

monotone if $M\left(x_{1}, y_{1}\right) \leq M\left(x_{2}, y_{2}\right)$ for $x_{1} \leq x_{2}, y_{1} \leq y_{2}$,

homogeneous if $M(\lambda x, \lambda y)=\lambda M(x, y)$ for all $x, y, \lambda>0$.

Note that since there are no decreasing means, a "monotone mean" means in fact an "increasing mean".

Classical means will be denoted by sans-serif capital letters. Thus

$$
\begin{aligned}
& \mathrm{A}(x, y)=\frac{x+y}{2}, \quad \mathrm{G}(x, y)=\sqrt{x y}, \quad \mathrm{H}(x, y)=\frac{2 x y}{x+y}, \\
& \mathrm{~L}(x, y)=\frac{x-y}{\log x-\log y}, \quad \mathrm{P}_{1}(x, y)=x, \quad \mathrm{P}_{2}(x, y)=y
\end{aligned}
$$

denote respectively the arithmetic, geometric, harmonic, logarithmic and the two projective means. For historical reason the exception is made for minimum and maximum means that are denoted by min and max.

If $F: \mathbb{R}_{+}^{2} \rightarrow \mathbb{R}_{+}$is homogeneous (of the order 1 ), then $F(x, y)=y F\left(\frac{x}{y}, 1\right)$ for all $x, y>0$, so its values are uniquely determined by the trace function

$$
f:=F(\cdot, 1) \text {. }
$$


If $F$ is also symmetric, then the identity $F(x, 1)=x F\left(x^{-1}, 1\right)$ shows that it is uniquely determined by the restriction of the trace function $f$ to any of the intervals $[1, \infty)$ or $(0,1]$.

A homogeneous function $M$ is a mean if, and only if,

$$
0<\frac{m(x)-1}{x-1} \leq 1, \quad x>0, x \neq 1 ; \quad m(1)=1 ;
$$

moreover, if the trace function $m$ is increasing and $m(1)=1$, then $M$ is a mean.

The trace function will be denoted by corresponding lowercase letter.

\section{Generalized Projective Means}

In this section we construct $2^{\mathfrak{c}}$ pairs of symmetric means satisfying (1).

Let $\mathbb{X}=\mathbb{R}_{+}^{2} \backslash\left\{(x, x): x \in \mathbb{R}_{+}\right\}$. For $A \subset \mathbb{X}$ we define the generalized projective mean $\mathrm{P}_{A}: \mathbb{R}_{+}^{2} \rightarrow \mathbb{R}_{+}$by

$$
\mathrm{P}_{A}(x, y)= \begin{cases}x & (x, y) \in A, \\ y & (x, y) \notin A .\end{cases}
$$

A set $A \subset \mathbb{X}$ is called asymmetric if

$$
(x, y) \in A \Leftrightarrow(y, x) \notin A \quad \text { for } x \neq y .
$$

We denote $A^{\prime}=\mathbb{X} \backslash A$.

Note the following elementary properties of generalized projective means:

1. $\mathrm{P}_{1}=\mathrm{P}_{\mathbb{X}}, \mathrm{P}_{2}=\mathrm{P}_{\emptyset}$,

2. $\min =\mathrm{P}_{\{(x, y): x<y\}}, \max =\mathrm{P}_{\{(x, y): x>y\}}$,

3. $\{x, y\}=\left\{\mathrm{P}_{A}(x, y), \mathrm{P}_{A^{\prime}}(x, y)\right\}$,

4. $\mathrm{P}_{A}$ is symmetric if and only if $A$ is asymmetric,

5. $\mathrm{P}_{A}$ is homogeneous if and only if $A$ is a positive cone, i.e. $A=\lambda A$ for all $\lambda>0$.

The property 3 implies that we can replace the means in (1) by

$$
K_{t, A}=t \mathrm{P}_{A}^{t}(x, y) \frac{x-y}{x^{t}-y^{t}} \quad \text { and } \quad L_{t, A}=t \mathrm{P}_{A^{\prime}}^{t}(x, y) \frac{x-y}{x^{t}-y^{t}}
$$

preserving the invariance property. Playing with parameter $A$ according to properties 4 and 5, one obtains symmetric or/and homogeneous solutions. Later we will construct a lot of other solutions to the invariance problem.

\section{The $L$ is Not Enough}

In this section we follow the ideas developed in [3] to obtain complementary means for other means than the logarithmic one. Given symmetric, homogeneous mean $M$ we shall seek for a pair of means of the form $x^{t} M_{t}^{1-t}(x, y)$, $y^{t} M_{t}^{1-t}(x, y)$, where $-1<t<1$. Let us give it a try. 


$$
M\left(x^{t} M_{t}^{1-t}(x, y), y^{t} M_{t}^{1-t}(x, y)\right)=M_{t}^{1-t}(x, y) M\left(x^{t}, y^{t}\right)=M(x, y) .
$$

Solving this equation for $M_{t}$ we obtain

$$
M_{t}(x, y)=\left(\frac{M(x, y)}{M\left(x^{t}, y^{t}\right)}\right)^{\frac{1}{1-t}}
$$

If $M_{t}$ happens to be a mean and $t>0$, then obviously both $\mathrm{P}_{1}^{t} M_{t}^{1-t}$ and $\mathrm{P}_{2}^{t} M_{t}^{1-t}$ are means and satisfy $M \circ\left(\mathrm{P}_{1}^{t} M_{t}^{1-t}, \mathrm{P}_{2}^{t} M_{t}^{1-t}\right)=M$. They remain means for $t<0$, due to the identity $x^{t} M_{t}^{1-t}=y^{-t} M_{-t}^{1+t}$.

The following theorem gives a simple criterion for $M_{t}$ to be means.

Lemma 1. If $M$ is a homogeneous, symmetric mean, then the conditions are equivalent:

(a) For every real $-1<t<1$ the function

$$
M_{t}(x, y)=\left(\frac{M(x, y)}{M\left(x^{t}, y^{t}\right)}\right)^{\frac{1}{1-t}}
$$

is a mean.

(b) $M$ is monotone.

Proof. Suppose $M$ is monotone. Then for $x<y$ we have

$$
x^{1-t}=\frac{M\left(x^{t} x^{1-t}, y^{t} x^{1-t}\right)}{M\left(x^{t}, y^{t}\right)} \leq \frac{M(x, y)}{M\left(x^{t}, y^{t}\right)} \leq \frac{M\left(x^{t} y^{1-t}, y^{t} y^{1-t}\right)}{M\left(x^{t}, y^{t}\right)}=y^{1-t},
$$

so $M_{t}$ is a mean.

Assume (a) holds. Since $M$ is homogeneous and symmetric, it suffices to show that its trace $m$ increases. If $x \leq 1 \leq y$, then setting $t=0$ we see that $m(x) \leq 1 \leq m(y)$. If $1<x<y$ then there exists $0<t<1$ such that $x=y^{t}$ and then

$$
1 \leq m_{t}^{1-t}(y)=\frac{m(y)}{m(x)},
$$

which concludes the proof, since the case $x<y<1$ is similar.

So we have proved the following fact.

Theorem 1. If $M$ is a monotone, homogeneous and symmetric mean and for $-1<t<1$ the functions $M_{t}$ are given by (3), then

$$
K_{t}(x, y)=x^{t} M_{t}^{1-t}(x, y) \quad \text { and } \quad L_{t}(x, y)=y^{t} M_{t}^{1-t}(x, y)
$$

are homogeneous means and satisfy

$$
M \circ\left(K_{t}, L_{t}\right)=M .
$$

Arguing as in the previous section we obtain the corollary. 
Corollary 1. If $M$ is a monotone, symmetric and homogeneous mean, and $M_{t}$ is given by formula (3), then for any generalized projective mean $\mathrm{P}_{A}$ the functions

$$
K_{t, A}(x, y)=\mathrm{P}_{A}^{t}(x, y) M_{t}^{1-t}(x, y) \quad \text { and } \quad L_{t, A}(x, y)=\mathrm{P}_{A^{\prime}}^{t}(x, y) M_{t}^{1-t}(x, y)
$$

are homogeneous means and satisfy

$$
M \circ\left(K_{t, A}, L_{t, A}\right)=M .
$$

Note that the means $K_{t}$ and $L_{t}$ inherit the symmetry and homogeneity, but in general, the monotonicity is not hereditary.

Theorem 2. If $M \neq \max , K_{t}$ and $L_{t}$ are as in Theorem 1 and

$$
\lim _{x \rightarrow 0+} m(x)>0,
$$

then the means $K_{t}$ and $L_{t}$ are not monotone if $t>0$.

Proof. Consider the mean $L_{t}$. One has

$$
\lim _{x \rightarrow 0+} l_{t}(x)=\lim _{x \rightarrow 0+} m_{t}^{1-t}(x)=1=l_{t}(1),
$$

which shows that $l_{t}$ is not monotone. Case $K_{t}$ is similar.

Note that in case of the logarithmic mean the functions obtained are weighted geometric means of a projective mean and a Stolarsky mean STO $1, t$. The Stolarsky means defined (in general case) by

$$
\operatorname{STO}_{r, s}(x, y)=\left(\frac{s}{r} \frac{x^{r}-y^{r}}{x^{s}-y^{s}}\right)^{\frac{1}{r-s}}=\left(\frac{\mathrm{L}\left(x^{r}, y^{r}\right)}{\mathrm{L}\left(x^{s}, y^{s}\right)}\right)^{\frac{1}{r-s}}
$$

are monotone, and therefore the resulting means (1) are so. But the Stolarsky means contain a group of means for which the resulting invariant means lack monotonicity. These are all $\mathrm{STO}_{r, s}$ with $r, s>0$, in particular the arithmetic mean $\mathrm{A}=\mathrm{STO}_{2,1}$ and the generalization of the Heronian means

$$
\mathrm{STO}_{1+\frac{1}{n}, \frac{1}{n}}(x, y)=\frac{x+x^{\frac{n-1}{n}} y^{\frac{1}{n}}+\cdots+x^{\frac{1}{n}} y^{\frac{n-1}{n}}+y}{n+1} .
$$

All of them have a non-zero limit at zero, thus the resulting means are not monotone.

The reader will verify that if $M \circ(K, L)=M$ and $M(0+, 1)>0$ and $K(0+, 1)=0$, then $L$ is not monotone.

\section{One More Step Towards Invariance}

The generalized projective means are a kind of extremities in the world of means. It is natural to ask whether similar solution can be obtained for arbitrary means. Let us formulate the problem as follows. 
Problem 1. Suppose $M$ is a symmetric, homogeneous and increasing mean and $t>0$. Characterize the means $C, D$ for which there exists a bivariate function $N$ such that both $C^{t} N^{1-t}$ and $D^{t} N^{1-t}$ are means and

$$
M \circ\left(C^{t} N^{1-t}, D^{t} N^{1-t}\right)=M .
$$

One can easily calculate that

$$
N(x, y)=N_{t}(x, y)=\left(\frac{M(x, y)}{M\left(C^{t}(x, y), D^{t}(x, y)\right)}\right)^{\frac{1}{1-t}} .
$$

The answer depends very much on the means involved. Here are some examples.

Example 1. For $t<\frac{1}{2}$ and arbitrary means $C, D$

$$
\left(\frac{\mathrm{G}}{\mathrm{G} \circ\left(C^{t}, D^{t}\right)}\right)^{\frac{1}{1-t}}
$$

is a mean.

Indeed, since $\frac{\mathrm{G}^{2}}{K}$ is a mean for arbitrary mean $K$, and $\mathrm{G}^{\frac{1}{t}} \circ\left(C^{t}, D^{t}\right)$ is a mean, we can write

$$
\left(\frac{\mathrm{G}}{\mathrm{G} \circ\left(C^{t}, D^{t}\right)}\right)^{\frac{1}{1-t}}=\left(\frac{\mathrm{G}^{2}}{\mathrm{G}^{1 / t} \circ\left(C^{t}, D^{t}\right)}\right)^{\frac{t}{1-t}} \mathrm{G}^{1-\frac{t}{1-t}}
$$

so the left-hand side is a weighted geometric mean of means.

Example 2. For arbitrary $0<t<1$ the function

$$
\left(\frac{\mathrm{A}(x, y)}{\mathrm{A}\left(\mathrm{A}^{t}(x, y), \mathrm{H}^{t}(x, y)\right)}\right)^{\frac{1}{1-t}}
$$

is not a mean.

Suppose for $x>1$ the inequality

$$
\left(\frac{\mathrm{a}(x)}{\mathrm{A}\left(\mathrm{a}^{t}(x), \mathrm{h}^{t}(x)\right)}\right)^{\frac{1}{1-t}} \leq x
$$

is valid. This is equivalent to

$$
\frac{a(x)}{x} \leq \frac{a^{t}(x)+h^{t}(x)}{2 x^{t}} .
$$

As $x$ tends to infinity, the left-hand side tends to $\frac{1}{2}$, while the limit of the right-hand side is $\frac{1}{2^{1+t}}$, so the assumption was wrong.

This example can be generalized.

Example 3. If $M, K, L$ are homogeneous means satisfying

$$
\lim _{x \rightarrow 0+} m(x)>0, \quad \lim _{x \rightarrow \infty} \frac{l(x)}{k(x)}=0, \quad \lim _{x \rightarrow \infty} \frac{k(x)}{x}<1,
$$


and $M$ is symmetric, then for any $0<t<1$ the corresponding function is not a mean, because

$$
\begin{aligned}
\lim _{x \rightarrow \infty} \frac{1}{x}\left(\frac{m(x)}{M\left(k^{t}(x), l^{t}(x)\right)}\right)^{\frac{1}{1-t}} & =\lim _{x \rightarrow \infty} \frac{1}{x}\left(\frac{x m\left(\frac{1}{x}\right)}{k^{t}(x) m\left(\frac{l^{t}(x)}{k^{t}(x)}\right)}\right)^{\frac{1}{1-t}} \\
& =\lim _{x \rightarrow \infty}\left(\frac{x}{k(x)}\right)^{\frac{t}{1-t}}>1 .
\end{aligned}
$$

One can easily verify, that for $p>0$ the functions $\mathrm{STO}_{1, p}, \mathrm{~A}$ and $\mathrm{H}$ satisfy the conditions in Example 3.

Given the fact, that $\lim _{p \rightarrow 0+} \mathrm{STO}_{1, p}=\mathrm{L}$ pointwise, the next example looks a little bit surprising.

Example 4. The function

$$
N(x, y)=\left(\frac{\mathrm{L}(x, y)}{\mathrm{L}\left(\mathrm{A}^{1 / 2}(x, y), \mathrm{H}^{1 / 2}(x, y)\right)}\right)^{2}
$$

is a mean.

To show this we need some quite elementary facts. First note

$$
1<\frac{\mathrm{a}(x)}{\mathrm{h}(x)}<x \quad \text { for } x>1 .
$$

By the convexity of $\sinh$ in $\mathbb{R}_{+}$, the divided difference $\frac{\sinh u}{u}$ increases. Hence, taking into account that

$$
\mathrm{L}\left(x, x^{-1}\right)=\frac{\sinh \log x}{\log x}
$$

we conclude that the function $(1, \infty) \ni x \mapsto \mathrm{L}\left(x, x^{-1}\right)$ is positive and increases.

To show that $N$ is a mean it is enough to prove that for $x>1$ the inequalities $1<N(x, 1)=n(x)<x$ hold. The left one is valid, because $\sqrt{\mathrm{a}(x) \mathrm{h}(x)}=\sqrt{x},(6)$ and (5) yield

$$
\begin{aligned}
\sqrt{n(x)} & =\frac{\mathrm{l}(x)}{\mathrm{L}(\sqrt{\mathrm{a}(x)}, \sqrt{\mathrm{h}(x)})} \\
& =\frac{\sqrt{x} \mathrm{~L}\left(\sqrt{x}, \frac{1}{\sqrt{x}}\right)}{\sqrt[4]{\mathrm{a}(x) \mathrm{h}(x)} \mathrm{L}\left(\sqrt[4]{\frac{\mathrm{a}(x)}{\mathrm{h}(x)}}, \sqrt[4]{\frac{\mathrm{h}(x)}{\mathrm{a}(x)}}\right)}>\sqrt[4]{x}>1 .
\end{aligned}
$$

To show the other inequality remind that the power mean of order $1 / 2$

$$
\mathrm{A}_{1 / 2}(x, y)=\left(\frac{\sqrt{x}+\sqrt{y}}{2}\right)^{2}=\left(\frac{\mathrm{L}(x, y)}{\mathrm{L}(\sqrt{x}, \sqrt{y})}\right)^{2}
$$


satisfies $A_{1 / 2} \leq A$. Then we use (5) and the monotonicity of the logarithmic mean to obtain

$$
\begin{aligned}
\sqrt{n(x)} & =\frac{\mathrm{I}(x)}{\mathrm{L}(\sqrt{\mathrm{a}(x)}, \sqrt{\mathrm{h}(x)})}=\frac{1}{\sqrt{\mathrm{a}(x)}} \frac{\mathrm{I}(x)}{\mathrm{I}\left(\sqrt{\frac{\mathrm{h}(x)}{\mathrm{a}(x)}}\right)} \\
& <\frac{1}{\sqrt{\mathrm{a}(x)}} \frac{\mathrm{I}(x)}{\mathrm{I}\left(\frac{1}{\sqrt{x}}\right)}=\sqrt{\frac{\mathrm{a}_{1 / 2}(x)}{\mathrm{a}(x)}} \sqrt{x}<\sqrt{x} .
\end{aligned}
$$

The above examples show that to decide whether a particular function is a mean might be quite complicated. But in fact, we do not need that much. Fortunately we can prove that the functions $C^{t} N^{1-t}$ and $D^{t} N^{1-t}$ are means.

Theorem 3. If $M$ is a symmetric, homogeneous, increasing mean and $C$ and $D$ are arbitrary means, then for all $0<t<1$ the functions

$$
K_{t}(x, y)=C^{t}(x, y) N_{t}^{1-t}(x, y) \quad \text { and } \quad L_{t}(x, y)=D^{t}(x, y) N_{t}^{1-t}(x, y),
$$

where $N_{t}$ is given by (4), are means and satisfy the invariance equation

$$
M\left(K_{t}(x, y), L_{t}(x, y)\right)=M(x, y) .
$$

Proof. Take arbitrary $x, y>0, x<y$. Our goal is to show that

$$
x \leq C^{t}(x, y) N_{t}^{1-t}(x, y) \leq y \quad \text { and } \quad x \leq D^{t}(x, y) N_{t}^{1-t}(x, y) \leq y .
$$

We shall prove the left inequalities, the proof of the right ones being similar. Note firstly that

$$
C^{t}(x, y) N_{t}^{1-t}(x, y)=\frac{M(x, y)}{M\left(\frac{D^{t}(x, y)}{C^{t}(x, y)}, 1\right)}
$$

and consider two cases:

CASE $C(x, y) \leq D(x, y)$.

In this case

$$
1 \leq \frac{D^{t}(x, y)}{C^{t}(x, y)} \leq \frac{D(x, y)}{C(x, y)} \leq \frac{y}{x}
$$

and

$$
M\left(\frac{D^{t}(x, y)}{C^{t}(x, y)}, 1\right)>1
$$

so

$$
y \geq M(x, y) \geq \frac{M(x, y)}{M\left(\frac{D^{t}(x, y)}{C^{t}(x, y)}, 1\right)} \geq \frac{M(x, y)}{M\left(\frac{y}{x}, 1\right)}=x .
$$

CAse $C(x, y)>D(x, y)$.

Now

$$
1 \geq \frac{D^{t}(x, y)}{C^{t}(x, y)} \geq \frac{D(x, y)}{C(x, y)} \geq \frac{x}{y}
$$


and

So

$$
M\left(\frac{D^{t}(x, y)}{C^{t}(x, y)}, 1\right)<1
$$

$$
x \leq M(x, y) \leq \frac{M(x, y)}{M\left(\frac{D^{t}(x, y)}{C^{t}(x, y)}, 1\right)} \leq \frac{M(x, y)}{M\left(\frac{x}{y}, 1\right)}=y
$$

Clearly, the means $K_{t}$ and $L_{t}$ inherit the symmetry and/or homogeneity from $C$ and $D$. The discussion on monotonicity from the previous section applies here as well.

The method described above works very well in case of monotone, symmetric and homogeneous means $M$. Clearly, homogeneity is crucial here, but one can ask whether the monotonicity condition can be relaxed?

Open Question 1. Consider a symmetric and homogeneous mean M. Do there exist three functions $K, L, N$ and a real number $0<t<1$ such that the functions $K^{t} N^{1-t}$ and $L^{t} N^{1-t}$ are means and the equality

$$
M\left(K^{t} N^{1-t}, L^{t} N^{1-t}\right)=M
$$

holds?

The examples in Sect. 5 show that the next question may be challenging as well.

Open Question 2. Suppose $M$ is a symmetric, homogeneous and increasing mean. Do there exist non-trivial means $C, D$ and a real number $0<t<1$ such that the function

$$
N(x, y)=\left(\frac{M(x, y)}{M\left(C^{t}(x, y), D^{t}(x, y)\right)}\right)^{\frac{1}{1-t}}
$$

is a mean?

\section{Means of $\boldsymbol{n}$ Variables}

It is natural to ask, whether Theorem 3 can be extended to higher dimension. Assume then $n>2$ and let $M, C_{1}, \ldots, C_{n}: \mathbb{R}_{+}^{n} \rightarrow \mathbb{R}_{+}$be means with $M$ symmetric, homogeneous and increasing. For arbitrary $t \in(0,1)$ the invariance equation

$$
M \circ\left(C_{1}^{t} N_{t}^{1-t}, \ldots, C_{n}^{t} N_{t}^{1-t}\right)=M
$$

can be solved in the same way as in case of means of two variables. So we obtain

$$
N_{t}=\left(\frac{M}{M \circ\left(C_{1}^{t}, \ldots, C_{n}^{t}\right)}\right)^{\frac{1}{1-t}}
$$


The example below shows the answer to our question is negative.

Example 5. Take $M=C_{1}=\mathrm{A}, C_{2}=\cdots=C_{n}=\mathrm{G}$ and let $t \in(0,1)$ be arbitrary. Then the function

$$
K_{t}=\mathrm{A}^{t} \frac{\mathrm{A}}{\mathrm{A} \circ\left(\mathrm{A}^{t}, \mathrm{G}^{t}, \ldots, \mathrm{G}^{t}\right)}
$$

is not a mean.

Indeed, setting $x_{1}=1$ and $x_{2}=\cdots=x_{n}=x$ we obtain

$$
\begin{aligned}
\lim _{x \rightarrow \infty} \frac{K_{t}(1, x, \ldots, x)}{\max (1, x, \ldots, x)} \\
=\lim _{x \rightarrow \infty} \frac{\left(\frac{1+(n-1) x}{n}\right)^{t}}{x} \frac{1+(n-1) x}{\left(\frac{1+(n-1) x}{n}\right)^{t}+(n-1)\left(\sqrt[n]{x^{n-1}}\right)^{t}} \\
=\lim _{x \rightarrow \infty} \frac{\frac{1}{x}+(n-1)}{1+(n-1)\left(\frac{x^{n-1}}{\left(\frac{1}{n}(1+(n-1) x)\right)^{n}}\right)^{\frac{t}{n}}}=n-1>1 .
\end{aligned}
$$

We want to emphasize that even if the functions $C_{i}^{t}(\mathbf{x}) N_{t}^{1-t}(\mathbf{x})$ may not be means, they form an $n$-tuple of invariant functions - a fact that can be useful in some applications.

\section{Remark on Translative Means}

A mean $N: \mathbb{R}^{2} \rightarrow \mathbb{R}$ is called translative if $N(x+\tau, y+\tau)=N(x, y)+\tau$ for all $\tau, x, y \in \mathbb{R}$.

Recall that a bivariate mean on $\mathbb{R}^{2}$ is both homogeneous and translative if, and only if, it is a weighted arithmetic mean [1, Theorem 1, p. 234]. This fact explains great popularity of the arithmetic means. As an example of a translative mean one can recall the Toader mean [10] defined as $E(x, y)=$ $\frac{x e^{x}-y e^{y}}{e^{x}-e^{y}}$.

Since a mean $M: \mathbb{R}_{+}^{2} \rightarrow \mathbb{R}_{+}$is homogeneous if, and only if, the mean $N: \mathbb{R}^{2} \rightarrow \mathbb{R}$ defined by

$$
N(x, y):=\log M(\exp x, \exp y),
$$

is translative, all the notions, results and questions posed above have their "translative" counterparts. In particular Theorem 1 can be reformulated as follows.

Theorem 4. If $N: \mathbb{R}^{2} \rightarrow \mathbb{R}$ is a monotone, translative and symmetric mean and for $-1<t<1$ the functions $N_{t}$ are given by

$$
N_{t}(x, y)=\frac{N(x, y)-N(t x, t y)}{1-t}, \quad x, y \in \mathbb{R}
$$

then the functions 


$$
K_{t}(x, y)=t x+(1-t) N_{t}(x, y) \quad \text { and } \quad L_{t}(x, y)=t y+(1-t) N_{t}(x, y)
$$

are translative means and satisfy

$$
N \circ\left(K_{t}, L_{t}\right)=N \text {. }
$$

Example 6. Since the arithmetic mean $\mathrm{A}$ is monotone, translative and symmetric, applying this result we conclude that $\mathrm{A} \circ\left(K_{t}, L_{t}\right)=\mathrm{A}$, that $\mathrm{A}$ is invariant with respect to the mean-type mapping $\left(K_{t}, L_{t}\right)$, where

$$
K_{t}(x, y)=\frac{1+t}{2} x+\frac{1-t}{2} y \quad \text { and } \quad L_{t}(x, y)=\frac{1-t}{2} x+\frac{1+t}{2} y
$$

for $x, y \in \mathbb{R}$.

It is not difficult to check that $\left\{\left(K_{t}, L_{t}\right): t \in(-1,1)\right\}$ is a family of all weighted arithmetic mean-type mapping such that $\mathrm{A} \circ\left(K_{t}, L_{t}\right)=\mathrm{A}$.

\section{Remarks on Subtranslative and Subhomogeneous Means}

Let $p \in \mathbb{R}$ be fixed. It is obvious that every function $N:(0, \infty)^{2} \rightarrow \mathbb{R}$ satisfying the inequality

$$
N(t x, t y) \leq t^{p} N(x, y), \quad x, y, t>0,
$$

is homogeneous of the order $p$, that is

$$
N(t x, t y)=t^{p} N(x, y), \quad x, y, t>0 .
$$

In this connection the following notion is natural. A mean $N:(0, \infty)^{2} \rightarrow$ $(0, \infty)$ is called subhomogeneous (see $[9])$, if

$$
N(t x, t y) \leq t N(x, y), \quad x, y>0, t>1 ;
$$

and superhomogeneous, if the reversed inequality holds true.

Analogously, a mean $N: \mathbb{R}^{2} \rightarrow \mathbb{R}$ is said to be subtranslative if

$$
N(x+t, y+t) \leq N(x, y)+t, \quad x, y \in \mathbb{R}, t>0
$$

and supertranslative if the reversed inequality is satisfied (cf. also Sándor [8] where translativity is called additive homogeneity).

Remark 1. Let $M, K, L:(0, \infty)^{2} \rightarrow(0, \infty)$ be means such that $M$ is homogeneous, strictly monotonic and invariant with respect to the mean-type map ( $K, L)$, that is $M \circ(K, L)=M$. If $K$ is subhomogeneous (superhomogeneous), then $L$ is superhomogeneous (subhomogeneous).

Proof. The $(K, L)$-invariance and homogeneity of $M$ yield

$$
M(K(t x, t y), L(t x, t y))=t M(x, y), \quad x, y>0, t>1 .
$$

Assuming that $K$ is subhomogeneous, i.e. that $K(t x, t y) \leq t K(x, y)$ for all $x, y>0$ and $t>1$, from the monotonicity of $M$, we hence get

$$
M(t K(x, y), L(t x, t y)) \geq t M(x, y), \quad x, y>0, t>1
$$


whence, by the homogeneity of $M$,

$$
M\left(K(x, y), \frac{1}{t} L(t x, t y)\right) \geq M(x, y), \quad x, y>0, t>1 .
$$

Now this inequality, the invariance equality

$$
M(K(x, y), L(x, y))=M(x, y), \quad x, y>0,
$$

and the increasing monotonicity of $M$ imply that

$$
\frac{1}{t} L(t x, t y) \geq L(x, y), \quad x, y>0, t>1,
$$

which proves that $L$ is superhomogeneous.

We omit a obvious reformulation of this remark in the case when $M$ is translative, $K$ and $L$ are sub- or super-translative.

Example 7. Put $L:=\mathrm{L}$ (the logarithmic mean) and $K:=2 \mathrm{~A}-\mathrm{L}$. Since $\mathrm{A}$ is translative, $\mathrm{L}$ is supertranslative (cf. Sándor [8]), and of course,

$$
\mathrm{A} \circ(K, \mathrm{~L})=\mathrm{A},
$$

that is $\mathrm{A}$ is $(K, \mathrm{~L})$-invariant, it follows that $K$ is subtranslative.

Open Access. This article is distributed under the terms of the Creative Commons Attribution 4.0 International License (http://creativecommons.org/licenses/ by/4.0/), which permits unrestricted use, distribution, and reproduction in any medium, provided you give appropriate credit to the original author(s) and the source, provide a link to the Creative Commons license, and indicate if changes were made.

\section{References}

[1] Aczél, J.: Lectures on Functional Equations and Their Applications. Academic Press, New York (1966)

[2] Gauss, C.F.: Bestimmung der Anziehung eines elliptischen Ringen. Ostwalds Klassiker Exakt. Wiss. Akademische Verlagsgesellschaft, Leipzig (1927)

[3] Kahlig, P., Matkowski, J.: Logarithmic complementary means and an extension of Carlson's log. Math. Inequal. Appl. 18(3), 1143-1150 (2015)

[4] Matkowski, J.: Iterations of mean-type mappings and invariant means. Ann. Math. Silesianae 13, 211-226 (1999)

[5] Matkowski, J.: Invariant and complementary means. Aequ. Math. 57, 87107 (1999)

[6] Matkowski, J.: Iterations of the mean-type mappings (ECIT' 08). In: Sharkovsky, A.N., Susko, I.M. (eds.) Grazer Math. Ber., Bericht Nr. 354, pp. 158-179 (2009)

[7] Matkowski, J.: Iterations of the mean-type mappings and uniqueness of invariant means. Ann. Univ. Sci. Bp. Sect. Comput. 41, 145-158 (2013)

[8] Sándor, J.: On certain subhomogeneous means. Octogon Math. Mag. 8(1), 156$160(2000)$ 
[9] Sándor, J., Toader, Gh.: On some exponential means. In: Seminar on Mathematical Analysis, Preprint vol. 3, pp. 35-40. Babeş-Bolyai University, Cluj (1990)

[10] Toader, Gh.: An exponential mean. In: Seminar on Mathematical Analysis, Preprint vol. 5, pp. 51-54. Babeş-Bolyai University, Cluj (1988)

[11] Witkowski, A.: On Seiffert-like means. J. Math. Inequal. 9(4), 1071-1092 (2015)

[12] Witkowski, A.: On two- and four-parameter families. RGMIA 12(1), Article 3 (2009)

Janusz Matkowski

Faculty of Mathematics

Computer Sciences and Econometrics

University of Zielona Gora

Szafrana 4A

65-516 Zielona Góra

Poland

e-mail: J.Matkowski@wmie.uz.zgora.pl

Monika Nowicka and Alfred Witkowski

Institute of Mathematics and Physics

UTP University of Science and Technology

al. prof. Kaliskiego 7

85-796 Bydgoszcz

Poland

e-mail: monika.nowicka@utp.edu.pl;

alfred.witkowski@utp.edu.pl

Received: August 12, 2015.

Accepted: December 30, 2015. 